\title{
Cardiorenal versus renocardiac syndrome: is there a difference?
}

\author{
Robert W Schrier
}

Many epidemiological observations have been made of a correlation between cardiovascular morbidity and mortality and decreased kidney function. This relationship exists regardless of whether the initial event is cardiac disease or renal parenchymal disease. The cardiovascular mortality of patients with congestive heart failure whose serum creatinine level is only moderately elevated (e.g. by $26.5 \mu \mathrm{mol} / \mathrm{I}$ $[0.3 \mathrm{mg} / \mathrm{dl}])$ has been shown to be increased (Dries D et al. [2000] J Am Coll Cardiol 35: 781-792). The mechanisms underlying this 'cardiorenal syndrome' have not been clearly elucidated. Nevertheless, increased cardiac preload and cardiac dilatation are known to be associated with enhanced ventricular wall stress, cardiac remodeling, increased left ventricular mass index and higher mortality (Supplementary Figure 1 online; Schrier RW [2006] J Am Coll Cardiol 47: 1-8).

As the kidney is the primary regulator of sodium and water excretion, even a modest decrease in normal renal function in patients with congestive heart failure could contribute to increased cardiac preload, cardiac dilatation, left ventricular hypertrophy and increased mortality. This sequence of events can, therefore, justifiably be termed 'cardiorenal syndrome'.

Renal parenchymal disease is also associated with an increase in the risk of cardiovascular death. Again, elevation of serum creatinine concentration by as little as $26.5 \mu \mathrm{mol} / \mathrm{l}$
In contrast to cardiorenal syndrome.... should the enhancement of cardiovascular death initiated by kidney

disease

be termed

'renocardiac syndrome'?

$R W$ Schrier is Editor-in-Chief of Nature Clinical Practice Nephrology.

Competing interests The author declared no competing interests.

www.nature.com/clinicalpractice doi:10.1038/ncpneph0673
$(0.3 \mathrm{mg} / \mathrm{dl})$ can increase this risk. In fact, $90 \%$ of patients with chronic kidney disease will die of cardiovascular complications before they progress to end-stage renal disease. The processes underlying the increase in cardiovascular mortality initiated by primary kidney disease are not well defined, but there are several potential mechanisms. These include, but are not limited to, uncontrolled hypertension, phosphate retention, secondary hyperparathyroidism, myocardial and vascular calcification, inflammation and oxidant injury (Supplementary Figure 2 online; Go A et al. [2004] N Engl J Med 351: 1296-1305).

In contrast to cardiorenal syndrome, in which the initiating event is heart disease, should the enhancement of cardiovascular death initiated by kidney disease be termed 'renocardiac syndrome'? Is this merely a semantic issue, or should cardiologists and nephrologists collaborate to understand and intervene in these two distinct syndromes, which account for a large proportion of cardiovascular deaths worldwide? Lastly, it should be acknowledged that there is overlap between these two syndromes; diabetes and hypertension can initiate both cardiac and renal pathology.

Supplementary information in the form of two figures is available on the Nature Clinical Practice Nephrology website. 